\title{
Austrian Dental Technician Award 2016
}

\section{Mit diesem Preis sollen zahntechnische Arbeiten ausgezeichnet werden}

Die Bundesinnung der Zahntechniker hat in einer Sitzung Anfang des Jahres das Angebot des Österreichischen Dentalverbandes den Austrian Dental Technician Award auszuloben, einstimmig angenommen.

Mit diesem Preis sollen zahntechnische Arbeiten ausgezeichnet werden.

Dem Österreichischen Dentalverband war diese Initiative wichtig, um auch auf diesem Weg zu zeigen, dass die Zahntechnikerinnen und Zahntechniker für die Dental- industrie und den Dentalhandel ein wichtiger Kundenkreis sind.

In den Gesprächen, welche im Anschluss an die Grundsatzentscheidung folgten, wurden die Ausschreibungsrichtlinien formuliert (Link siehe unten).

Parallel hierzu wurden die Sponsoren für den ADTA 2016 gesucht. Der Hauptpreis wird vom Österreichischen Dentalverband gesponsert. Die weiteren Sponsoren sind - in alphabetischer Reihung Dental Journal, Henry Schein Dental Austria GmbH, Ivoclar Vivadent $\mathrm{GmbH}$, Pluradent
Austria GmbH, Wieladent und ZPP Dentalmedizintechnik $\mathrm{GmbH}$.

Ausschreibung:

http://www.odv.dental/wpcontent/uploads/2015/11/ Ausschreibungsrichtlinien_ ADTA2016_NEU.pdf

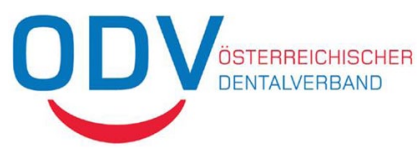

Achtung, die Termine für Einreichung der Arbeiten und Vergabe der Preise werden auf 2017 verschoben. Die neuen Termine werden noch bekannt gegeben.

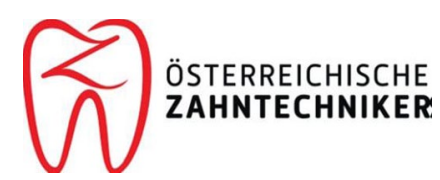

Stomatologie 2015 - 112:316 DOI 10.1007/s00715-015-0082-1 Online publiziert: 4. Dezember 2015 ๑) Springer-Verlag Wien 2015 